\title{
Emerging Scenario on Industrial Use of Bio Resources and Development of Models for Computing Access and Benefit Sharing (ABS) Mechanisms
}

Anantha Narayana $\mathrm{DB}^{1^{*}}$ and Sudhakar Johnson $\mathrm{T}^{2}$

${ }^{1}$ Chairman, Herbs and Herbal Products Committee of Scientific body of Indian Pharmacopoeia Commission former, Member of Scientific Panel of Food Safety and Standards Authority of India (FSSAl), New Delhi, India

${ }^{2}$ Department of Biotechnology and Center for Innovation, Incubation \&Entrepreneurship, K. L. University, Green Fields, Vaddeswaram, Guntur Dt. 522502, A. P. India

"Corresponding author: Anantha Narayana DB, Chairman, Herbs and Herbal Products Committee of Scientific body of Indian Pharmacopoeia Commission former, Member of Scientific Panel of Food Safety and Standards Authority of India (FSSAI), New Delhi, India, Tel: +91-080-26420775; E-mail: dba.narayana@gmail.com

Received Date: July 10, 2017; Accepted Date: July 19, 2017; Published Date: July 26, 2017

Copyright: ( 2017 Narayana DBA. This is an open-access article distributed under the terms of the Creative Commons Attribution License, which permits unrestricted use, distribution, and reproduction in any medium, provided the original author and source are credited.

Views Expressed in this Paper are not of the Organizations to which the authors are affiliated to

\begin{abstract}
Objectives: Bio resources are sources for major economies in the world and provide livelihood opportunities for local tribes. Bio resources form part of raw material for herbal products / medicine manufacturing industry. One of the objectives of the Convention on Biological Diversity (CBD) is access and benefit sharing (ABS) arising out of utilization of bio resources. ABS calculation is most debated issue among end user - herbal products / medicine manufacturing industry.
\end{abstract}

Methods and Results: Mechanisms for ABS computation are still evolving in many nations. In order to remove disparity in ABS calculation and bring in transparency, in this article we have presented three models. In the process, we have developed bio resource sustainability grid to map bio resources based on the i) ease of access, ii) regenerability of resources and iii) sustainability. First model describes calculation of ABS according to cost of bio resources, the second model links to proportion of bio resource with proportion of final formulation and the third model provides a formula which is analogous to Drug Price Control Order.

Conclusion: With increase in use of botanical dietary supplements, nutraceuticals and emerging research in phytopharmaceuticals as drugs the quantum of usage of bio resources will proportionally increase. In addition to usage, conservation of critical bioresources is responsibility of stakeholders. The ABS computation approaches we have presented here can provide transparent ABS computation. The models can be adopted by other nations BDAs either directly or after minor modifications. The purpose of transparency in ABS calculation is served as both the end user and regulator can calculate the ABS payments without debate. Further, it would supplement the cause of sustainable utilization of bioresources while promoting conservation.

Keywords: Access and benefit sharing; Biodiversity; Biological diversity act; CBD; Herbal industry; Sustainable utilization

Abbreviations ABS: Access and Benefit Sharing; BDA: Biodiversity Act; BR: Bioresources; CBD: Convention on Biological Diversity; CoP: Conference of Parties; DPCO: Drug Price Control Order; NBA: National Biodiversity Authority

\section{Background}

The biological resources are sources of many major economies in the world and livelihood options for rural, remote tribes. Biological resources are used in food, fuel, cosmetics, pharmaceuticals, traditional medicines, nutraceutical industries, to name a few [1]. In addition to above, bio resources have wider applications such as in paper manufacturing, timber, spices, oleoresins, aromatic oils. It is now, more or less accepted by end users that unsustainable utilization without conservation will not only affect the economy, rural livelihood, species survival but also the climate $[1,2]$. Thus it is important to conserve biological resources and responsibility lies both with society and its end users. Herbal products/medicine manufacturing industries are major end users. Although bio resources have wider applicability, in this article, we have restricted the context to herbal medicine and herbal extract manufacturing industry.

The Convention on Biological Diversity (CBD) [3] has three objectives, viz., the conservation of biological diversity, the sustainable use of its components and the fair and equitable sharing of the benefits arising out of the utilization of genetic resources. The ratification of CBD in the year 1993 brought the importance of access and benefit sharing (ABS) The Nagoya protocol on ABS [4] under the CBD access to genetic resources and the fair and equitable sharing of benefits - was adopted by tenth CoP (Conference of Parties). Subsequently many countries have formulated their own policies to successfully implement the objectives. Calculation of access and benefit sharing has however become the most contentious issue. Existing rules of ABS varied from country to country and calculations are considered arbitrarily by many users and stake holders. In the absence of uniform calculation mechanism, arriving at the sharing of the benefits of bio resource utilization is debated issue. End users argue that benefit sharing should be based on quantity of bio resources that goes into final formulation. Further, it is argued that benefit sharing should be different for renewable bio resources and most difficult to access nonrenewable resources. Benefit sharing need to be different where only 
access to the bio resource is involved and where bio resource is accessed along with knowledge associated with it. In order to address these challenges and to remove disparities while bringing in logical computing mechanisms are necessary. In this article, we propose three approaches for access and benefit sharing calculations. In the process we have developed a simple bio resource sustainability grid to map renewable, accessibility and sustainable nature of bio resources. While developing the approaches we have kept in consideration of i) quantity of bio resources used in the final product, ii) the sustainable nature and iii) accessibility of bio resources iv) nature and extent of associated knowledge accessed. The proposed models offer potential approaches for consideration by countries in addition to India in their respective ABS calculation mechanisms. The ABS calculations are referred here restricted to monetary benefits and do not deal with non-monetary contributions to ABS which are permitted in the biodiversity regulations. Further, it is clearly indicated that use of bio resource for isolation of an API (Active Pharmaceutical Ingredient) which can become an NCE (New Chemical Entity) and get developed into a drug is out of the purview of present article. The proposed models are applicable for bio resources in its raw form or processed form including standardized extracts /distillates/expressed oils etc. to obtain a finished product with intended use as a traditional medicine/herbal product/cosmetic/supplements.

\section{Indian biodiversity act evolution and brief note on benefit sharing}

It is notable that India was among the first few countries that enacted legislation on ABS to fulfill its national obligations under CBD. India enacted a Biodiversity Act (BDA) in the year 2002 (BDA 2002) as per which the National Biodiversity Authority (NBA) of India was established. The (BDA) is in line with $\mathrm{CBD}$, putting emphasis on regulating access of bio resources by organizations or individuals who are not Indian citizens. Local communities and traditional healers who use bio resources are exempted under the Act from ABS. However, the rules are also stringent for Indian herbal product / medicine industries. $\mathrm{ABS}$ is one of the most contentious issues felt by herbal industry users, especially the large traditional medicine manufacturing industry which used many forms of bio resources. The industry utilizes medicinal plants, their parts, processed medicinal plants as raw material for decades in their pharmacies and manufacturing industry. Herbal extracts industry is also one of the major users of bio resources. It is reasonable to anticipate that the industry demanded clarity of applicability of BDA with respect to ABS to bio resources and sharing of benefits. When the BDA regulation was passed in 2002 several concerns remained unanswered, such as legal status and ownership of genetic resources, lack of clarity on commonly held bio resources, etc., The Government of India's new guidelines notified in 2014 [5,6] have provided certain provisions to the existing regulations related to ABS. The BDA has been demanding ABS payments from the traditional medicine industry of Indian origin for last few years while most of them do not specifically come under section 3 (Clause 3 (2), Chapter 2 of BDA, 2002) which refers to individuals or organizations covered under the act. In this context it is to be recognized that most of the traditional medicine industry is an outcome of and result of scaling up of traditional practices followed by Vaidyas (traditional doctors) and other traditional healers, who are legally permitted to practice their profession. During such practice historically they were either collecting bioresources or procuring them from traders, collectors and tribals. Such bioresources were used to produce the traditional medicines. Many of them believe that scaling up of activity to larger batch sizes in the factories is result of their entrepreneurial capability and hence as per the exemption clauses they are not covered to meet ABS requirements. Further it was felt that they are, as per rules under BDA, only required to intimate the information related to bio resources used by them to the State Biodiversity Boards (Clause 7, Chapter 2 of BDA, 2002).

\section{BDA in other nations}

Several other nations have put forth BDA guidelines in place. We have presented a few such guidelines and their ABS models. Brazilian biodiversity (new framework approved by Senate on April 7, 2015) [7] insists that the Companies have to register electronically in order to access genetic resources. The new regulation ensures traceability of the product chain. Benefit sharing agreement must be executed after product development. In case of access to genetic resources beneficiary is a fund set up by government and in case of access to traditional knowledge the beneficiary are the communities. In case of access to genetic resources, the manufacturer of product must share $1 \%$ of annual net sales. Similar to Indian biodiversity rules, Brazilian rules exempt from benefit sharing obligations to traditional farmers, unions in addition to micro and small enterprises and individual micro entrepreneurs. It also exempts the intermediate producer, not responsible for the finished product.

Bhutanese biodiversity rules [8] allow access to applicant after mutual agreement and if one or more following minimum conditions are met for benefit sharing: a flat fee and upfront payment, the sharing of research results, royalties, milestone payments, recognition as a partner in intellectual property ownership of products, free supply of commercial products derived from the accessed resources

The Ministry of Natural Resources and Environment (MNRE) of Malaysia [9] is a competent authority to access to genetic resources or traditional knowledge. All access requires permit, regardless of whether the access is for commercial or non-commercial use. Noncommercial access must be done in conjunction with a domestic institution, while commercial applicants must establish fair and equitable benefit sharing. South Africa's NEMBA (National Environmental Management Biodiversity Act) [10] categorized bio prospecting in two phases, viz., discovery phase and commercialization phase. According to section 81 (1) of these regulations a person may not, without a permit, engage in commercialization phase of bio prospecting involving indigenous genetic biological resources (IGBR) or export IGBRs. Similar to Indian and Brazilian rule, NEMBA protects access provider (a person, a community or organ of state) and a traditional knowledge (TK) holder (indigenous community or individual). The regulations apply to commercial or individual sectors that uses IGBRs/ and or TK for commercial activities and non-commercial sector that export IGBRs from South Africa (SA) for research to generate data. The SA regulations require that the applicant for a permit be either a corporation registered under SA law, or a permanent resident of SA. If the applicant is a foreign or Corporation they must apply jointly with a SA corporation or citizen/ permanent resident Overall, we have noticed that many countries' access and benefit sharing rules are still evolving

\section{Methods}

As a part of the policy development, the authors have met and discussed formally and informally, with various stakeholders, viz., 
Associations of herbal and Ayurvedic drug manufacturers, herbal raw material supply chain professionals, herbal regulatory experts, scientific community. Much of these discussions have spread over several years. The contents of this paper are the results of such discussions put together.

Such interactions have been in the form of bringing greater clarity and add value to the basic tenets of the models developed by the authors. Herbal medicine industry uses mainly two types of categories, the first one being the crude bio resources (medicinal plants and the parts in their unprocessed or minimally processed forms) as their input raw materials. Minimally processed means - drying after collection in air or sun, sorting to remove foreign matter / adulterants, washing and drying, cutting to manageable sizes, and such unit operations which do not alter the structure and permits identification of the bio resources. The broad uses of bio resources are mostly limited to medicinal plants / botanicals. The list of usage of bio resources are provided in Figure 1. A brief diagrammatic sketch providing the journey of bio resources from the source to the user industry is given in Figure 2.

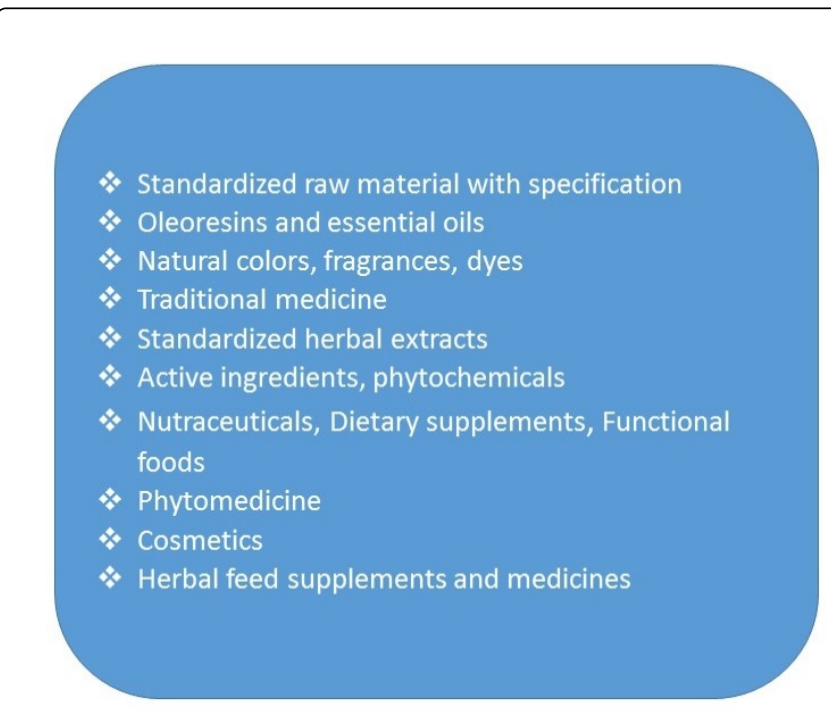

Figure 1: Broad listing of uses of bioresources in an herbal industry. List of value added products from bioresources in a typical herbal industry. The products range from simple crude bioresources (medicinal plants and the parts in their unprocessed or minimally processed forms) to high end value added forms such as phytomedicines, fractionated extracts, purified compounds, nutraceuticals and dietary supplements. Value added forms fetch premium price apart from reducing packing and transportation cost. The proportion of bioresources in a final formulation may vary from less than $1 \%$ to as much as $95 \%$. ABS calculation should be based on the proportion of bioresources used in the formulation.

The second category of bio resources are the raw material that are converted to value added products namely - extracts, fractionated extracts, purified compounds isolated from the raw material, oleoresins, distilled / vegetable oils, gums, starches, salts and ester forms of these and other processed materials. Such processed materials in most cases are value added products as they reduce the quantity of use (instead of large proportions of minimally processed raw materials), and fetch premium price apart from reducing packing and transportation cost. Such processed materials may themselves be used as supplements, nutraceuticals, botanical drugs, Ayurveda drugs, additives in pharmaceuticals and food formulations. Many processed materials also find a place in cosmetic formulations. The above formulations may contain anywhere from very low levels (say less than $1 \%$ of the bio resource in the final formulations) going up to as much as $95 \%$ content.

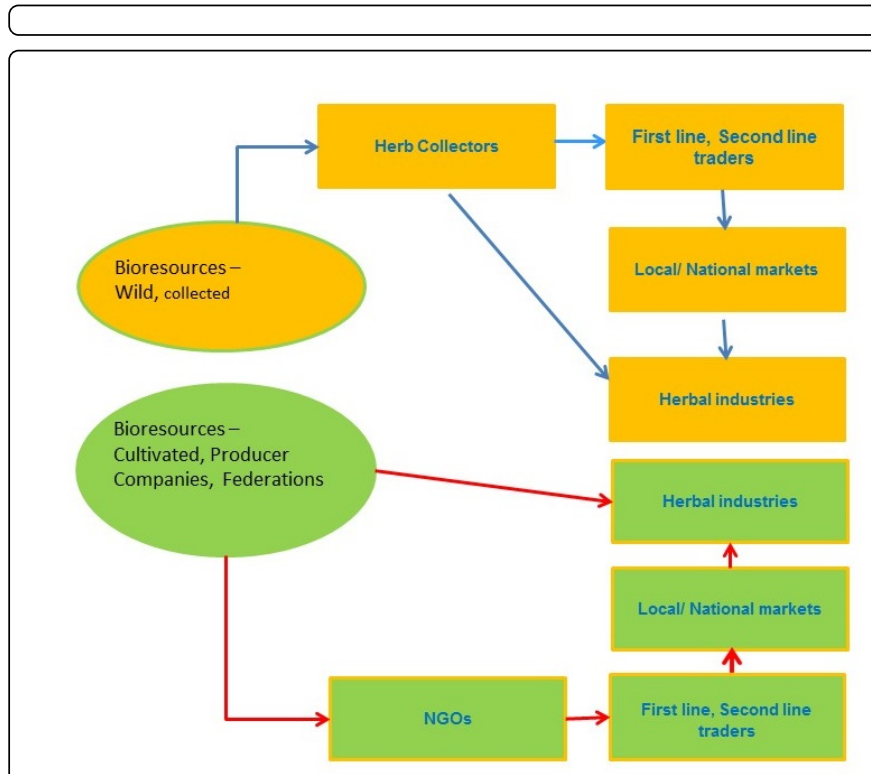

Figure 2: Mapping the journey of bioresources till the industrial use. The detailed listing or traceability of bioresources. The bioresources are either collected from forests or cultivated in the agriculture farms to meet the demand of herbal industry. Cultivation of bioresources under the guidance of forest department, federations or group of self-help farmers is recommended as issues such as utilization and conservation are considered.

Since herbal medicine industry uses bio resources in huge quantity, the industry representatives, associations have been requesting and demanding the authorities to clarify that for value added products of bioresources the regulations of ABS do not apply as per their interpretations. However, the necessary clarifications have not been forthcoming from the concerned authorities. Many members of the industry have in the last one decade or so initiated and invested in backward integration of medicinal plants by getting them cultivated in part of agricultural lands. The sector is of the opinion that bioresources obtained from such cultivated sources should be out of the purview of $\mathrm{ABS}$ regulations

The methods also involve a short review of available literature, and the insights of the authors to this sector having been a part of the same for many years, and does not involve any data generation on quantum of bio resources usage or the ABS amounts. 


\section{Results and Discussion}

\section{Development of bio resource sustainability grid to calculate ABS based on physical access to bio resources}

Guidelines on access and benefit sharing: We present here one typical example of access and benefit sharing as per Indian BDA rules.

\begin{tabular}{|c|c|c|}
\hline Condition & Payment by trader & Payment by manufacturer \\
\hline $\begin{array}{l}\text { Where no prior benefit sharing (BS) agreement with joint } \\
\text { forest } \\
\text { management committee (JFMC)/ } \\
\text { gram sabha/forest dweller/ cultivar }\end{array}$ & $1 \%-3 \%$ of the purchase price & $3 \%-5 \%$ of purchase price \\
\hline $\begin{array}{l}\text { Further sale of biological resource by a trader to another } \\
\text { trader/ manufacturer }\end{array}$ & $\begin{array}{l}1 \%-3 \% \text { of the purchase price (in case there is proof } \\
\text { of supply chain then BS only on amount for which BS } \\
\text { has not been paid earlier) }\end{array}$ & $3 \%-5 \%$ of purchase price \\
\hline $\begin{array}{l}\text { Where there is prior BS agreement with JFMC/ gram } \\
\text { sabha/forest price dweller/ cultivar }\end{array}$ & Not less than $3 \%$ of purchase price & Not less than $5 \%$ of purchase \\
\hline High economic value bio resource such as Red sanders & $\begin{array}{l}\text { Upfront payment of not less than } 5 \% \text { of } 5.0 \% \text { on the } \\
\text { proceeds of the auction, or sale amount, as decided } \\
\text { by the NBA or SBB into a designated fund }\end{array}$ & $\begin{array}{l}\text { Upfront payment of not less than } 5 \% \text { of } 5.0 \% \text { on } \\
\text { the proceeds of the auction, or sale amount, as } \\
\text { decided by the NBA or SBB into a designated } \\
\text { fund }\end{array}$ \\
\hline $\begin{array}{l}\text { Where access leads to commercial utilization, optional } \\
\text { benefit on ex-factory sale price }\end{array}$ & $\begin{array}{l}\text { Rs.0.1 million (0.1\%); Rs. } 0.1-0.3 \text { million }(0.2 \%) \\
\text { above } 0.3 \text { million }(0.5 \%)\end{array}$ & $\begin{array}{l}\text { Rs. } 0.1 \text { million }(0.1 \%) \text {; Rs } 0.1-0.3 \text { million }(0.2 \%) \text {; } \\
\text { above Rs. } 0.3 \text { million }(0.5 \%)\end{array}$ \\
\hline
\end{tabular}

Table 1: Access and benefit sharing guidelines issued by National Biodiversity Authority. Abbreviations used: JFM: Joint Forest Management; NBA: National Biodiversity Authority; SBB: State Biodiversity Board.

Current biodiversity rules for access and benefit sharing for commercial usage of bio resources is briefly described in Table 1 [6].

Other aspects of ABS such as access for transfer of research results, access for intellectual property rights and transfer of research results for research/commercial utilization were not discussed in this article.

For the purpose of developing ABS computation model, we have first generated bio resources sustainability grid (Figure 3).

Based on the availability, access, regenerability and sustainability nature bio resources have been categorized into four quadrants (Q1 to Q4). The parts of the plant accessed make it either easy or difficult to regenerate. Root, bark, sapwood, heartwood etc., fall into category of difficult to regenerate and destructive harvesting while regenerable parts such as leaves, stem of annuals, twigs, regenerate quickly when they are removed or clipped form the plants. Bio resources that are easy to regenerate, easy to access and abundantly available are placed in Q1 (Serial number 1-4). Examples include Andrographis paniculata (serial number 1 of Q1). In the same quadrant the plant Woodfordia fruticosa whose flowers are utilized are placed in top most part of Q1 indicating tough access. Similarly, in Q2 fruits and flowers of climbers, epidermal hairs that have difficulty in access and renewability (Serial number 5-9) are placed. Bio resources categorized in quadrant Q3 are difficult to access, difficult to regenerate and less abundant (serial number 10-12). Bio resources in Q4 are most difficult to regenerate, most difficult to access and rare. For example, plants that are listed in CITES-I and II, and plants with endangered status and bio resources that are prone to destructive harvest are categorized in Q4 (serial number 13-18). These are only representative examples, however, exceptions can be found. In this approach when BR's are accessed physically without any associated knowledge, it is proposed that computation of ABS be linked to the cost of raw bio resource (unprocessed or minimally processed) at which the BR's are procured by the user industry and to be paid to BDA while procuring the BR. The quantum of such payment is in relation to the position of the BR in this proposed grid. This would offer a transparent and logical frame work to the industry linked to sustainability.

\section{Approaches proposed for computation of ABS based on access to associated knowledge}

The grid discussed in Figure 3 deals with physical access to bio resources. The authors are proposing yet another mechanism of computation of ABS based on associated knowledge accessed. The mechanism considers a) proportion of bio resources used in final formulation and $\mathrm{b}$ ) access to associated traditional knowledge (Figure 4).

For e.g., during preparation of a product, existing traditional knowledge used will have certain fee of ABS. Further, if the inventor has developed or added new knowledge that is not existing hitherto, inventor need not pay the same amount or pay a reduced fee in the form of ABS. The Indian herbal medicine industry recognizes the need for conservation of bioresources and do not argue against the same. However, the sector feels that they are exempt from $\mathrm{ABS}$ requirements. The sector wherever required to comply with $\mathrm{ABS}$ requirements demands: i) monetary payments of ABS where required should be based on simple and transparent system of computation, ii) such 
payments should be based on the nature of bioresource accessed and the proportion of bioresource going into the formulation. Greater quantum the use greater efforts needed for conservation and renewal of the bioresources and hence proportionate higher payment, iii) the system should recognize that mere addition of a bioresoruces into a formulation does not necessarily contribute fully to the commercial success of the formulation. Many times especially in case of cosmetics, the bioresourcess form a low level of usage and mostly for varianting purposes. Marketing and sales investments including brand building expenses go into making the formulation succeed in the market apart from investments in R\&D and innovations. Linking the ABS payment and quantum of payment to either retail price of formulation or sales turnover of formulation or profit of the company or from the product is not logical.

\section{Figure-3}

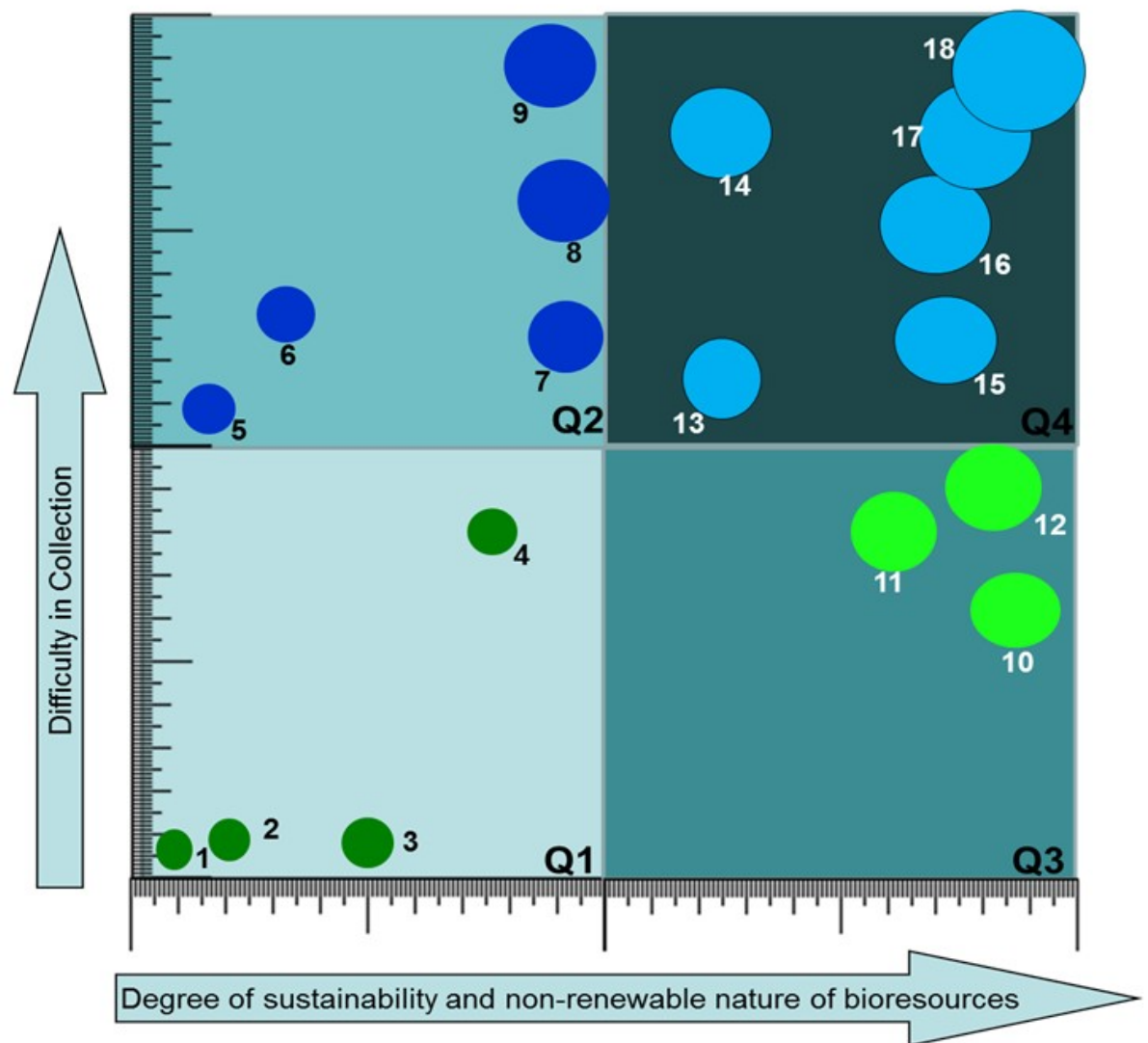

Figure 3: Development of bio resource sustainability Grid No. 1. Biodiversity sustainable grid is developed for the purpose of developing ABS computation model. Based on the availability, access to bioresources, regenerability and sustainability, bioresources were grouped into four quadrants (Q1 to Q4). 1: Leaves from small trees/shrubs (Murayya koenigi), 2: Leaves from bushes (Adathoda vasika), 3: Annuals (Andrographis paniculata), 4: Flowers (Woodfordia fruticose), 5: Leaves from climbers (Gymnema sylvestris), 6: Fruits/flowers from trees (Aegle marmolas), 7: Fruit parts- epidermal hairs (Malalu philippinensis), 8: Gums/resins (Pinus roxburghii), 9: Galls (Pistacia integerrima), 10: Endemic annuals (Pimpinella tirupatensis), 11: Stem (Clerodendron phlomidis), 12: endemic wood tree (Pterocarpus santalinus), 13: Bark (Terminalia arjuna), 14: Lithophytes (Bergenia ligulata), 15: Rhizomes (Pueraria tuberosa), 16: Root/Root bark (Decalepsis hamiltoni), 17: CITES-I and II listed wild plants. Q1: Most easily accessible, renewable and sustainable (serial numbers 1-4); Q2: reasonably difficult to access and medium renewable (serial numbers 5-9); Q3: Difficult to collect and difficult to regenerate (serial numbers: 10 to 12); Q4: Most difficult to access and almost nil renewability and plants prone to destructive harvesting (serial numbers 13-18). The area or size of circle is inversely proportional to sustainability

Most product manufacturers would like to contribute through ABS payments based on the cost of raw materials accessed and used, iv) If the system still involves ABS payments linked to the formulation price or sales turnovers the system needs to recognize investments and expenses where inventive steps and innovations have been demonstrated and the system should provide some offset for this component from the ABS amount computed and v) the system should be logical and computable by both the regulator and the sector. If the system is at the point of access of the BR it would promote greater compliance. 
Use of Bio-resource (BR) or Associated Knowledge in a final formulation, Grid No.2

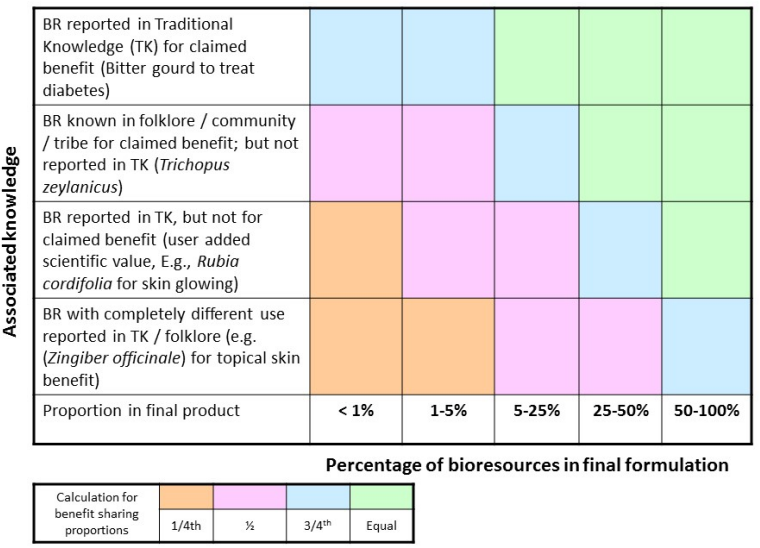

Figure 4: Development of Grid No.2 for computation of ABS. Development of Grid for computation of ABS when associated knowledge is accessed. Grid No. 2 ABS Computation also takes into account the percentage or proportion of bioresources used in the final formulation. The authors suggest that if an end user added inventive step to existing traditional knowledge such costs have to be take into consideration while computing ABS. For more details, refer to Model 2 of ABS computation. Color code represents percentage ABS to be paid to Biodiversity Authority.

Based on discussions with stake holders involved in the herbal manufacturing industry including those involved in conservation efforts and related organizations both formally and in informal discussions, the authors propose models to deal with ABS calculation and encourage development of harmonized system. The three proposed models are:

Model 1: Computation of the quantum of the cost of BR (minimally processed) based on a bio resources sustainability grid (Figure 3 ). The grid (Figure 3 ) has on its $\mathrm{X}$ axis "Degree of sustainability and nonrenewable nature of bio resources" and on $\mathrm{Y}$ axis "Difficulty in collection". As explained earlier in section 6 , the grid forms 4 quadrants and depending on the specific BR it would fall into one of the 4 quadrants. The model suggests fixing a percentage of cost of raw BR at which the industry buys the raw material for computation of the ABS to be paid by the accessor / user from industry. For example on a hypothetical case if the cost of raw BR is "US\$ N per kilogram" then $5 \%$ of $\mathrm{N}$ may be the quantum to be contributed to ABS fund as a onetime payment at the time of accessing. This $5 \%$ of $\mathrm{N}$ would apply to all BR's that fall into Q4 and 1/4th, 1/2, 3/4th of $5 \% \mathrm{~N}$ would apply to other quadrants, Q1, Q2 and Q3, respectively. The $5 \% \mathrm{~N}$ discussed here is only an example. Basis expertise on in-situ or ex-situ conservation efforts and costs, the percentage of $\mathrm{N}$ may be arrived by the authority in consultation with stake holders and notified. The above calculation highlights that those bio resources that are easy to regenerate would share lower ABS while those bio resources that are difficult to regenerate and are prone to destructive harvest share higher ABS.

Model 2: Computation of the quantum of ABS if it is to be linked to the final formulation (either its retail price or sales turnover or profit) is based on another grid (Figure 4). In this grid clear recognition and linkages to the proportion of use of BR in the formulation offered to consumers, existence or otherwise of any inventive steps and investments done in $\mathrm{R} \& \mathrm{D}$ over and above known information on the $\mathrm{BR}$ are given consideration. Here too, as in the grid shown in Figure 3, if $10 \% \mathrm{~N}$ is decided for ABS quantum, decisions on anywhere from $5 \%$ to $100 \%$ of $10 \% \mathrm{~N}$ to be applied to different grid blocks into which the access of bioresources and the associated information falls. Adoption of this grid would provide a transparent and simple system for computation of the quantum. It is easy to decipher the block to which the particular formulation case falls.

Model 3: Another approach could be to develop a computation formula similar to the one adopted by National Pharmaceutical Pricing Authority of India involved in Drug Price Control to compute maximum retail price of formulations [11] which is used to compute maximum retail prices of drug formulations. This formula termed as Drug Price Control Order (DPCO) [12] (Drug Price Control Order formula 2013) takes into consideration the raw material cost, packaging material cost, processing charges, and provides for a term "markup". This markup in case of drug pricing considers the commissions to be paid across the distribution chain, and profit to the manufacturer / marketer. The NPPA fixes the markup while deciding and notifying the maximum retail price of drug products which come under price control. This formula has built in component of excise duty. Drawing analogy of this formula to either a raw bioresource or a formulation containing bioresource, the quantum of amount to be charged as ABS (for conservation efforts of the particular BR) can be notified using the same term as "markup" or use a new term "ABS duty". For purposes of quick reference the formula under Drug Price Control Order is reproduced here.

Calculation of retail price of formulation (DPCO) is based on the following formula:

$$
\text { R.P }=(M . C+C . C+P . M+P . C) X(1+\mathrm{MAPE} / 100)+\mathrm{ED}
$$

Where, RP: retail price, MC: material cost (cost of drugs + excipients including overages), CC: conversion cost (worked out as per established procedure of costing and to be fixed by NPPA), PM: cost of packaging material (packaging cost including process losses to be fixed by NPPA), PC: packing charges (worked out as per established procedure of costing and to be fixed by NPPA), MAPE maximum allowable post- manufacturing expenses (all costs incurred by manufacturer from the stage of ex-factory cost to retailing, includes trade margin and margin for manufacturer - to be fixed by NPPA) and ED: excise duty.

On an analogy the DPCO formula if adopted for the context of ABS computation, it can be on lines below:

RP: retail price at which BR's are purchased by the industry, MC: material cost (cost of BR at the time of access), CC: conversion cost (expenses incurred for minimally processing explained in this article), PM: cost of packaging material (cost of packaging materials and packing ), PC: packing charges (expenses incurred for packing and transportation till the point of gate posts of forests / end user), MAPE (To be re-designated to ABS duty- includes margin to be paid to collector / packer / transporter/ traders and other stake holders in the supply chain + charges for conservation and maintenance of sustainability of the BR), ED: any duty or tax like "duty charged by forest official / octroi / sales tax etc. 


\section{Conclusion}

Several nations including India are striving to formulate bio diversity regulations to comply with international treaties and are facing growing pains in putting rules and systems in place. The pharmaceutical industry has over the years got the distinction of being a large supplier of drugs and pharmaceuticals and finished formulation. The Pharma sector is expected to see the next phase of growth with botanical drugs to be seen in future [13]. A paradigm shift from "ill centric "to "wellness centric" is driving towards growth in foods as medicine. The growth in use of dietary supplements, nutraceuticals [14] and emerging research in phyto pharmaceuticals as drugs will soon raise the quantum of usage of bio resources. The growing popularity of Ayurvedic products and traditional medicines is also putting pressure on the indigenous Ayurvedic industry which is witnessing higher demand for crude drugs. 'Back to nature' approach of consumers is further driving need for botanical, naturals, herbal extracts for use as supplements or cosmetic ingredients.

Hence the demand and supply situation for bio resources especially the medicinal plants, fruits and vegetables and other plant based raw materials will see drastic changes. Since bio diversity act and some of the rules there under are already in practice, clear and transparent policies and regulations need to be put in place to promote the herbal manufacturing industry in a sustainable way. Clear and transparent policy for applicability of access and benefit sharing (ABS) has to be in operation. The present article presented a few models in this direction. Adopting and evolving a workable formula on these lines in consultation with stake holders and regulators would be beneficial and consequently help promote development of the herbal medicine industry. It would supplement the cause of sustainable utilization of bioresources while promoting conservation. The models proposed here can be adopted by other nations BDAs either directly or after minor modifications. The purpose of transparency in ABS calculation is served as both the end user and regulator can calculate the ABS payments without debate.

\section{Acknowledgements}

Authors acknowledge the contributions of many scientists, supply chain executives, Ayurvedic industry personnel, herbal extracts industry personnel in India, with whom discussions took place informally and formally. Much of these discussions have spread over several years. These contributions have been in the form of bringing greater clarity and add value to the basic tenets of the models developed by the authors. TSJ thanks Head of the Department,
Biotechnology department, K L University and the University management for the support.

\section{Funding}

Part of funding was met by Authors own resources, since it is a policy/methodology article.

\section{Authors' contributions}

DBAN contributed to overall layout of the manuscript, regulatory related matters and calculation of three ABS models. TSJ contributed to bioresources and biodiversity discussion part. DBAN and TSJ together developed Grids 1 and 2 (i.e., Figures 3 and 4).

\section{References}

1. Johnson ST, Agarwal RK, Agarwal A (2013) Non-timber forest products as a source of livelihood option for forest dwellers role of society, herbal industries and government agencies. Curr Sci 104: 440-443.

2. Agarwal A, D'Souza P, Johnson ST, Dethe SM, Chandrasekaran CV (2014) Use of in vitro assays for assessing botanicals. Curr Op Biotechnol 25: 39-44.

3. (1993) Convention on Biological Diversity. UN Environment.

4. (2010) Nagoya protocol on Access and Benefit sharing. UN Environment

5. (2014) National Biodiversity Authority Notification. The gazette of India.

6. Wilson N (2015) Guidelines for access and benefit sharing for utilization of biological resources based on Nagoya protocol effective. J Intellectual Property Rights 20: 67-70.

7. No authors (2015) Brazilian biodiversity regulations. Community biodiversity management.

8. (2016) Bhutanese Government biodiversity regulations. Draft Biodiversity Bill.

9. (2016) Malaysia regulations. Tropical Forest Biodiversity Centre.

10. (2016) Bioprospecting regulatory framework guideline. South Africa regulation.

11. Deshpande SW, Gandhi N (2009) Drug Price Control Order, The Drugs and Cosmetics Act 1940 and Rules 1945, DPCO 1955, Susmit Publishers, Mumbai.

12. (2013) Drug price control Order formula. Ministry of Chemicals and Fertilizers.

13. Narayana DBA, Katiyar CK (2013) Draft amendment to drugs and cosmetics rules to license science based botanicals, phytopharmaceuticals as drugs in India. J Ayurveda Integr Med 4: 245-246.

14. (2015) Draft nutraceutical regulations, Food Safety and Standards Authority of India (FSSAI). 